\title{
Taxa de Gestação e Níveis Plasmáticos de Progesterona, em Receptoras de Embrião Bovino, Tratadas com Buserelina após a Inovulação ${ }^{1}$
}

\section{Antonio Marcos Galimberti ${ }^{2}$, Francisco Aloízio Fonseca ${ }^{3}$, Manoel Carlos Couto Araujo 4 , Eduardo Paulino da Costa ${ }^{5}$, Célio de Freitas ${ }^{6}$, José Domingos Guimarães ${ }^{5}$, Ademir de Moraes Ferreira ${ }^{6}$}

\begin{abstract}
RESUMO - Foram utilizadas 42 vacas ou novilhas mestiças, de aptidão leiteira, distribuídas em dois tratamentos: $\mathrm{T}_{1}(\mathrm{n}=22)$ - grupo controle, sem tratamento hormonal; $\mathrm{T}_{2}(\mathrm{n}=20)$ - grupo tratado com buserelina no quarto dia após a transferência de embrião (inovulação), com o objetivo de avaliar o efeito do tratamento hormonal na taxa de gestação e nas concentrações plasmáticas de progesterona das receptoras de embrião bovino. As coletas de sangue para obtenção do plasma sangüíneo foram realizadas no dia da inovulação (dia 0) e de dois em dois dias, num total de cinco amostras por receptora. O diagnóstico de gestação foi realizado via transretal. O uso de um agonista do GnRH (Buserelina) no $4^{\circ}$ dia após a inovulação não resultou em diferença entre os tratamentos na taxa de gestação. Entretanto, os níveis de progesterona das receptoras gestantes do grupo-tratado foram maiores em comparação às do grupo-controle.
\end{abstract}

Palavras-chave: bovino, buserelina, progesterona, taxa de gestação, transferência de embrião

\section{Pregnancy Rate and Plasma Progesterone Concentrations in Bovine Embryo Recipients Treated with Buserelin after Embryo Transfer}

\begin{abstract}
Forty-two crossbred dairy cows or heifers were randomly allocated to two treatments: $\mathrm{T}_{1}$ ( $\mathrm{n}=22$ ) - control group, without hormonal treatment; $T_{2}(n=20)$ - treated group with buserelin in the fourth day after embryo transfer, to evaluate the effect of hormonal treatment on the pregnancy rate and plasma concentrations of progesterone in bovine embryo recipients. Blood collection samples for plasma obtainment were realized on day of embryo transfer (day 0) and every other day, in a total of five samples per recipients. The pregnancy diagnosis was realized by transrectal way. The use of a GnRH agonist (buserelin) on the fourth day after embryo transfer did not result on difference between treatments in relation to the pregnancy rate. However, the plasma levels of progesterone of pregnant recipients of the treated group were greater as compared with those of the control group.
\end{abstract}

Key Words: bovine, buserelin, embryo transfer, progesterone, pregnancy rate

\section{Introdução}

Excluindo a falha na fertilização e perdas embrionárias antes de seis a oito dias, os fatores que determinam a perda ou a sobrevivência embrionária após a inseminação são similares àqueles que operam após a transferência de embriões para as receptoras (SREENAN e DISKIN, 1987). Segundo esses autores, a taxa de sobrevivência embrionária após a transferência de embriões pode ser influenciada por fatores como anormalidades cromossômicas, efeito da doadora, idade e qualidade dos embriões transferidos, método e local da transferência, sincronia doadora receptora, estado nutricional e níveis séricos de progesterona na receptora, bem como o estresse calórico (HANSEN e EALY, 1991).

Procurando otimizar os resultados da monta natural e da inseminação artificial, em vacas e/ou novilhas, alguns pesquisadores têm usado progestágeno (KESLER, 1997) ou agonistas do GnRH (NAKAO et al., 1983; MACMILLAN et al., 1986; STEVENSON et al., 1993; SHELDON e DOBSON, 1993; MANN e LAMMING, 1995). A morte embrionária no início da gestação contribui para redução na taxa de gestação de bovinos (SREENAN e DISKIN, 1987; MANN et al., 1995 e MANN e LAMMING, 1995). Os índices de 45 a $55 \%$ de taxa de gestação para transferência de embriões congelados (RODRIGUES,

\footnotetext{
${ }^{1}$ Parte do trabalho de tese de Mestrado apresentado pelo primeiro autor ao Departamento de Zootecnia da Universidade Federal de Viçosa, projeto financiado pela FAPEMIG.

2 Médico Veterinário, MSc, CRMV - ES no 358, Cx. Postal 037, 29.656-000, Alto Caldeirão, Santa Teresa - ES. E.mail: tonipat@ig.com.br

3 Professor do Laboratório de Zootecnia e Nutrição Animal - UENF, 28.015-620, Campos dos Goytacazes - RJ.

4 Doutorando em Medicina Veterinária, Escola de Veterinária, UFMG, 31.270-901, Belo Horizonte - MG.

5 Professor Adjunto do Departamento de Veterinária, UFV, 36.571-000, Viçosa - MG.

6 EMBRAPA Gado de Leite, Rua Eugênio do Nascimento, 610, Dom Bosco, 36.038-330, Juiz de Fora - MG.
} 
1992) e 62,5\% para não congelados (RUMPF et al., 1992) sugerem a necessidade de mais pesquisas para otimizar tais resultados.

O estabelecimento da gestação em vacas envolve complexa integração entre o embrião, ambiente uterino e o corpo lúteo (SREENAN e DISKIN, 1987; MANN et al., 1995; ROBERTS et al., 1996). Segundo MANN et al. (1995), o estabelecimento da gestação depende da produção do anti-luteolítico interferon trofoblástico pelo concepto em tempo hábil. A progesterona provoca modificações no ambiente uterino, influenciando o crescimento e desenvolvimento do concepto (GEISERT et al., 1992), mas, de acordo com MANN et al. (1995), o embrião pode não receber suficiente estímulo, mediado pela progesterona, para produção do interferon trofoblástico, necessário para bloquear o desenvolvimento do mecanismo luteolítico e manter a gestação.

O estádio fisiológico do útero pode ser modificado pela administração exógena de progesterona (LAWSON e CAHILL, 1983), haja vista o constante uso, por muitos pesquisadores, deste hormônio e/ou de substâncias luteotrópicas ou anti-luteolíticas, na tentativa de aumentar a taxa de sobrevivência embrionária (LEWIS et al., 1990; STEVENSON et al., 1993; RYAN et al., 1994).

O hormônio luteinizante apresenta maior capacidade de estimular a produção de progesterona pelo corpo lúteo (HOYER e NISWENDER, 1986; NISWENDER e NETT, 1988). A secreção de progesterona é mantida durante o início da gestação, principalmente pela ação dos fatores anti-luteolíticos que protegem o corpo lúteo do processo normal de luteólise (HAFEZ, 1992). A progesterona é importante no controle do mecanismo de luteólise, por inibir o desenvolvimento de receptores endometriais para a ocitocina (VALLET e LAMMING, 1991; LAU et al., 1992). Em vacas com baixa concentração de progesterona, esta inibição é menos efetiva (LAMMING e MANN, 1993) e o mecanismo luteolítico desenvolve-se antecipadamente, proporcionando menor tempo para o embrião produzir suficiente interferon trofoblástico capaz de bloquear a luteólise. REMSEM et al. (1982) encontraram correlação positiva entre a concentração de progesterona no dia da inovulação e a subsequente taxa de gestação, em um estudo com 223 receptoras de embriões bovinos.

A buserelina é um composto nonapeptídico agonista de $\mathrm{GnRH}$, com aproximadamente 40 vezes a potência do GnRH endógeno (STEVEN, 1983), capaz de alterar a função ovariana, modulando as ondas de crescimento folicular, prolongando a vida útil do corpo lúteo e a duração do ciclo estral (MILVAE et al., 1984; TWAGIRAMUNGU et al., 1992). O efeito primário do GnRH é estimular a síntese e a liberação do hormônio luteinizante (LH) da hipófise anterior, resultando também na síntese e/ou liberação, de forma menos pronunciada, do hormônio folículo estimulante (KALTENBACH et al., 1974; STEVEN, 1983).

A onda de LH induzida pelo GnRHe seus agonistas possui ação luteotrópica (RETTMER et al., 1992 e MANN et al., 1995). Estes pesquisadores verificaram aumento nos níveis séricos de progesterona, dois a três dias após a aplicação de buserelina. Também MILVAE et al. (1984) observaram prolongamento do ciclo e aumento dos níveis plasmáticos de progesterona, com a aplicação de buserelina entre os dias 9 a 12 após o estro, em vacas inseminadas.

Com o objetivo de avaliar os efeitos da aplicação única de buserelina, no quarto dia após a inovulação sobre a taxa de gestação e verificar as concentrações plasmáticas de progesterona em receptoras de embrião bovino, durante o período de reconhecimento materno da gestação na espécie bovina, foi desenvolvido este experimento.

\section{Material e Métodos}

O experimento foi conduzido no período de 10 de julho a 30 de novembro de 1997 na Fazenda Experimental Santa Mônica, de propriedade da EMBRAPA - Gado de Leite, localizada no município de Valença, RJ, de latitude $22^{\circ}$ - 21 e longitude $43^{\circ}$ - 42.

Foram utilizadas 42 vacas e/ou novilhas mestiças Holandês x Zebu como receptoras de embrião, com peso corporal igual ou superior a $350 \mathrm{~kg}$, que tenham apresentado atividade cíclica regular e estro sincronizado com prostaglandina $\mathrm{F}_{2} \alpha$ (Veteglan-luteolítico ${ }^{\circledR}$ - Serono Ltda), em função da data da coleta de embriões. Todas as receptoras foram examinadas por meio de vaginoscopia e palpação transretal, eliminando-se do programa de transferência de embriões as que apresentavam infeção uterina. As receptoras que receberam embriões estavam com escore corporal de 3 a 4 na escala de 1 a 5 (WILDMAN et al., 1982).

Foram utilizadas 16 vacas da raça Gir leiteiro, puras e registradas, como doadoras de embriões, as quais foram superovuladas com $400 \mathrm{mg}$ de hormônio folicular estimulante (FSH-P) de origem suína (Pluset - Serono Ltda), divididos em duas aplicações diárias a cada 12 horas, via intramuscular, em doses decres- 
centes, num total de oito aplicações durante quatro dias seguidos. O início do tratamento superovulatório ocorreu entre o oitavo e o décimo segundo dia do ciclo estral, considerando o dia do estro como dia 0 e coincidindo com a quarta e a quinta aplicação do FSH-P, foi administrado, via intramuscular, prostaglandina $\mathrm{F}_{2} \alpha$ (Veteglan-luteolítico ${ }^{\circledR}$ Serono Ltda) para indução do estro na doadora, na dose de $150 \mathrm{mg}$ de D + Cloprostenol em cada uma das duas aplicações.

Foram realizadas duas inseminações por doadora, utilizando-se sêmen de touro Holandês, sendo a primeira 12 horas após a doadora ter sido observada em estro, na situação de parada para monta, e a segunda 12 horas após a primeira.

As coletas de embriões foram realizadas no sétimo ou oitavo dias após o estro, por método não-cirúrgico, utilizando um cateter de foley conectado a um filtro com malha de 80 micrometros. O meio utilizado foi o PBS (Phosphate Bufered Saline) de Dulbecco e Vogt, modificado por WHITTINGHAM (1971), suplementado com $1 \%$ de soro fetal bovino inativado.

Após a coleta, com auxílio de microscópio estereoscópio (aumento de 10 a 40X), os embriões foram localizados, manipulados e avaliados, sendo classificados quanto ao estádio de desenvolvimento, segundo LINDNER e WRIGTH (1983), e ao aspecto morfológico, de acordo com KENNEDY et al. (1983). A transferência dos embriões foi realizada pelo método não-cirúrgico, no qual os embriões foram envasados em palhetas de $0,25 \mathrm{~mL}$ e depositados no corno uterino ipsilateral à ovulação, a fresco, com auxílio de aplicador e bainhas apropriadas.

As receptoras selecionadas foram distribuídas em um delineamento inteiramente casualizado com dois tratamentos sendo:

Tratamento 01 (Grupo-controle): composto de 22 receptoras, sendo 18 vacas e quatro novilhas, que receberam embriões transferíveis nos dias 6,7 ou 8 do ciclo estral $($ dia $0=$ dia do estro).

Tratamento 02 (Grupo-buserelina): composto de 20 receptoras, sendo 14 vacas e seis novilhas, que receberam embriões transferíveis nos dias 6,7 ou 8 do ciclo estral, e aplicação, via intramuscular, de $10 \mathrm{mg}$ de buserelina (Conceptal ${ }^{\circledR}$ - Hoechst) no quarto dia após a inovulação.

Amostras de sangue de cada receptora foram coletadas no dia da inovulação, e a cada dois dias até completar cinco amostras por receptora, sendo utilizados tubos vacuolizados com anti-coagulante heparina (Liquemine ${ }^{\circledR}$ ), pela punção da veia e/ou artéria coccígeas. Os tubos vacuolizados com as amostras foram acondicionadas em caixa de isopor contendo gelo, e em seguida centrifugados a $2500 \mathrm{rpm}$ durante 25 minutos; o plasma assim obtido foi envasado em tubetes de plástico com tampa, identificados com o número da receptora, bem como a data e o número de ordem da coleta de sangue, e conservados a $18^{\circ} \mathrm{C}$ até a análise hormonal.

As concentrações de progesterona foram dosadas pela técnica de radioimunoensaio (RIA), usando metodologia descrita no "kit" comercial (Coat - A Count ${ }^{\circledR}$ Progesterone) com procedimento em fase sólida, 125I (iodo radioativo), e contador de cintilações gama. As análises de progesterona foram realizadas no Laboratório de Biofísica e Fisiologia do Instituto de Ciências Biológicas da UFMG.

O diagnóstico de gestação foi realizado por meio de palpação transretal 45 dias após a inovulação.

Os resultados das análises de progesterona de cada grupo foram interpretados estatisticamente pelas análises de variância e regressão, e a taxa de gestação de cada tratamento foi interpretada estatisticamente pelo teste do qui-quadrado, segundo GOMES (1987).

\section{Resultados e Discussão}

No grupo-buserelina constituído de 20 receptoras, foram observadas nove vacas e quatro novilhas gestantes, em um total de 13 gestações $(65,0 \%)$ após tratamento com buserelina. No grupo-controle composto de 22 receptoras, foram observadas 11 vacas e três novilhas gestantes, num total de 14 gestações $(63,6 \%)$. Não houve diferença $(P>0,05)$ na taxa de gestação; os resultados estão apresentados no Tabela 1.

As taxas de gestação obtidas foram similares àquelas observadas por RYAN et al. (1994) e MANN et al. (1995), os quais utilizaram $10 \mathrm{mg}$ de buserelina, entre os dias 11 e 13 do ciclo estral (dia $0=$ dia do estro) de vacas e/ou novilhas, de rebanhos leiteiros comerciais que utilizavam a inseminação artificial. Da mesma forma, BECK et al. (1994) utilizaram $10 \mathrm{mg}$ de buserelina no dia 12 após a cobrição de ovelhas e não encontraram diferença na taxa de gestação. Portanto, o uso de um agonista do GnRH nesses casos não aumentou a taxa de gestação.

Estes resultados possivelmente podem ser atribuídos a um deficiente mecanismo anti-luteolítico entre o embrião e a unidade materna, uma vez que o embrião foi retirado do interior do útero da doadora com $7 \mathrm{ou}$ 8 dias e transferido para o interior uterino da receptora. Nesse sentido, FREI et al. (1989) realizaram estudo 
Tabela 1 - Número de receptoras de embrião bovino, gestantes e não-gestantes e porcentagem de gestação nos grupos tratado com $10 \mathrm{mg}$ de buserelina no $4^{\circ}$ dia após a inovulação e controle

Table 1 - Number of bovine embryo recipients, pregnants and non-pregnants and percentage of pregnancy in the treated group with $10 \mathrm{mg}$ of buserelin in the fourth day after embryo transfer and in the control group

\begin{tabular}{lcccc}
\hline $\begin{array}{l}\text { Grupos } \\
\text { Groups }\end{array}$ & $\mathrm{n}$ & $\begin{array}{c}\text { Receptoras gestantes } \\
\text { Pregnants recipients }\end{array}$ & $\begin{array}{c}\text { Receptoras não-gestantes } \\
\text { Non-pregnants recipients }\end{array}$ & $\begin{array}{c}\text { Porcentagem de gestação } \\
\text { Pregnancy rate }\end{array}$ \\
\hline $\begin{array}{l}\text { Tratado } \\
\text { Treated }\end{array}$ & 20 & 13 & 07 & 65,0 \\
$\begin{array}{l}\text { Controle } \\
\text { Control }\end{array}$ & 22 & 14 & 08 & 63,6 \\
\hline
\end{tabular}

qualitativo e quantitativo in vitro da síntese protéica, em embriões bovinos até o estádio de blastocisto, e observaram aumento dessa síntese entre 8 e 16 células (três a cinco dias) e também aumento progressivo na taxa de incorporação de metionina, na fase de 16 células até blastocisto ( 5 a 8 dias). Isto demonstra que o primeiro pico de síntese protéica ocorre ainda quando o embrião se encontra na doadora.

Caso as proteínas de origem embrionária do primeiro pico de síntese protéica (FREI et al., 1989), participem do início ou desencadeamento do mecanismo de reconhecimento materno da gestação in vivo, o fato de se coletarem os embriões do trato reprodutivo da doadora e transferi-los para o das receptoras poderia influenciar de forma negativa o mecanismo de reconhecimento materno da gestação, afetando a tecnologia de transferência de embriões em bovinos, por limitar os resultados desta técnica.

As concentrações plasmáticas de progesterona obtidas das fêmeas estão apresentadas no Tabela 2.

As concentrações plasmáticas de progesterona das receptoras não-gestantes do grupo-controle (Figura 1) seguiram ascendentes até o $6^{\circ}$ dia após a transferência de embriões, quando atingiram o pico, e a partir deste ponto houve declínio, o que pode ser devido à deficiência na produção de progesterona pelo corpo lúteo. Estes dados são semelhantes àqueles observados por MILVAE et al. (1984) e GONG et al. (1995) para vacas ou novilhas pertencentes ao grupo não-tratado nos respectivos experimentos.

Já os níveis plasmáticos de progesterona das receptoras não-gestantes do grupo-tratado (Buserelina) seguiram ascendente até o $4^{\circ}$ dia após a inovulação, quando atingiram o pico, e a partir deste ponto houve declínio na concentração de progesterona, sugerindo deficiência na produção de progesterona pelo corpo lúteo. Estes dados estão apresentados na Figura 1 e são similares aos observados por STEVENSON et al. (1993), em vacas inseminadas não-gestantes e tratadas com uma única dose de $8 \mathrm{mg}$ buserelina entre os dias 11 e 14 do ciclo estral.

Não houve diferença $(P>0,05)$ entre os níveis plasmáticos de progesterona das receptoras nãogestantes dos grupos tratado (Buserelina) e controle. RETTMER et al. (1992) também não observaram diferença entre as concentrações séricas de progesterona, em novilhas não-gestantes, tratadas ou não com $200 \mathrm{mg}$ de acetato de fertirelina entre os dias 11 e 13 do ciclo estral (dia $0=$ dia do estro).

Observou-se aumento linear nos níveis plasmáticos de progesterona, das receptoras gestan-

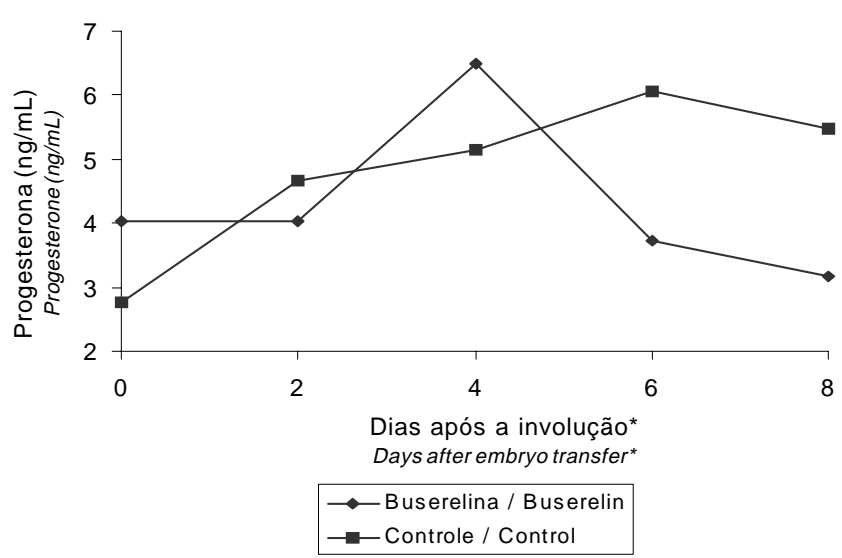

* Dia 0 = dia da inovulação.

* Day $0=$ day of the embryo transfer.

Figura 1 - Concentrações plasmáticas de progesterona nas receptoras de embrião bovino não-gestantes do grupo-controle e do grupo-tratado com $10 \mathrm{mg}$ de buserelina no $4^{\circ}$ dia após a inovulação.

Figure 1 - Plasma concentrations of progesterone on nonpregnants bovine embryo recipients of the control group and treated group with $10 \mathrm{mg}$ of buserelin in the fourth day after embryo transfer. 
Rev. bras. zootec.

Tabela 2 - Médias e desvios-padrão das concentrações plasmáticas de progesterona (ng/mL), nas receptoras de embrião bovino, gestantes e não-gestantes no grupo tratado com $10 \mathrm{mg}$ de buserelina no $4^{\circ}$ dia após a inovulação e controle

Table 2 - Means and standard deviations of the plasma progesterone concentrations $(\mathrm{ng} / \mathrm{mL})$ in pregnants and non-pregnants bovine embryo recipients in the treated group with $10 \mathrm{mg}$ of buserelin in the fourth day after embryo transfer and control group

\begin{tabular}{llr}
\hline $\begin{array}{l}\text { Componente } \\
\text { Component }\end{array}$ & Dias* & Progesterona $(\mathrm{ng} / \mathrm{mL}) \mathrm{Média} \pm$ D.P. \\
\hline
\end{tabular}

Grupo-tratado (Buserelina)

Treated group (Buserelin)

$\begin{array}{lcr} & 0 & 4,10 \pm 2,15 \\ \text { Receptoras gestantes } & 2 & 5,35 \pm 1,51 \\ \text { Pregnants recipients } & 4 & 6,13 \pm 1,68 \\ & 6 & 6,95 \pm 1,91 \\ \text { Média } & 8 & 7,51 \pm 2,32 \\ \text { Mean } & 6,01 \pm 2,23 & \\ & & 4,03 \pm 2,77 \\ \text { Receptoras não-gestantes } & 0 & 4,04 \pm 3,12 \\ \text { Non-pregnants recipients } & 2 & 6,48 \pm 4,59 \\ & 4 & 3,73 \pm 3,35 \\ \text { Media } & 6 & 3,17 \pm 3,11\end{array}$

Média

Mean

$4,29 \pm 3,44$

Grupo-controle

Control group

Receptoras gestantes

Pregnants recipients

Média

Mean

0

2

4

6

8

$5,29 \pm 1,94$

Receptoras não-gestantes

Non-pregnants recipients

Média

Mean

* Dia 0 = dia da inovulação.

DP = desvio-padrão.

* Day 0 = day of the embryo transfer.

$S D=$ standard deviation.

tes do grupo-controle (Figura 2). Este acréscimo foi significativo em função dos dias de coleta de sangue $(0,2,4,6$ e 8 ; sendo dia $0=$ dia da inovulação) e explicado pela equação linear $\mathrm{Y}=0,3668 \mathrm{x}+1,2596$ com $\mathrm{R}^{2}=0,92(\mathrm{P}<0,001)$.

Também foi observado aumento linear nos níveis plasmáticos de progesterona das receptoras gestantes do grupo-tratado (Buserelina, Figura 2). Este acréscimo foi significativo em função dos dias de coleta de sangue $(0,2,4,6$ e 8 ; sendo dia $0=$ dia da inovulação) e explicado pela equação linear $\mathrm{Y}=0,4215 \mathrm{x}$ $+1,3738$ com $\mathrm{R}^{2}=0,98(\mathrm{P}<0,0001)$.

A concentração plasmática de progesterona do grupo-tratado (Buserelina) foi maior $(\mathrm{P}<0,05)$ que os níveis plasmáticos deste hormônio apresentado pelo grupo-controle (Figura 2).

Observações similares foram realizadas por STEVENSON et al. (1993), que utilizaram $8 \mathrm{mg}$ de buserelina, entre os dias 11 e 14 do ciclo estral de vacas leiteiras inseminadas (dia $0=$ dia do estro), e similares também aos dados obtidos por RYAN et al. (1994), que utilizaram $10 \mathrm{mg}$ de buserelina, no dia 12 após a inseminação artificial de vacas leiteiras (dia 0 = dia do estro).

HARVEY et al. (1994) e MANN e LAMMING (1995) sugerem que o aumento nos níveis séricos de progesterona em vacas tratadas com $10 \mathrm{mg}$ de buserelina, entre os dias 11 e 13 do ciclo estral, é 


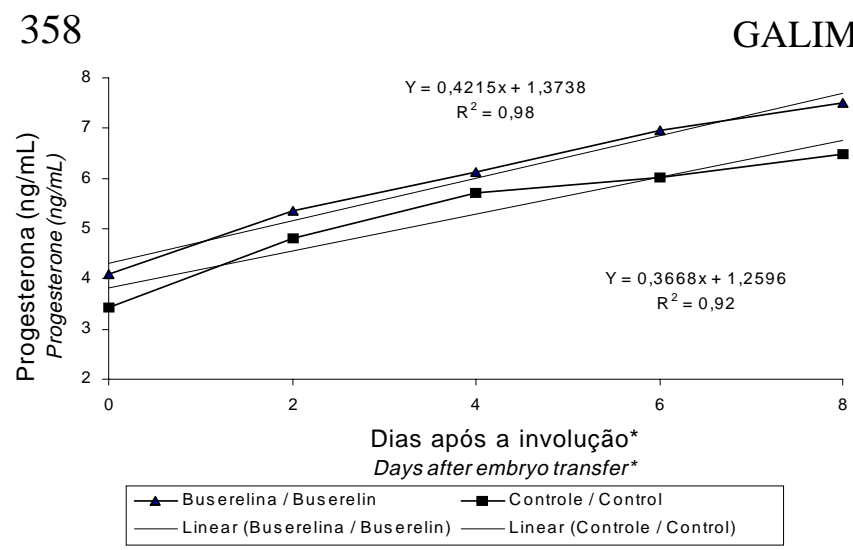

* Dia $0=$ dia da inovulação.

${ }^{*}$ Day $0=$ day of the embryo transfer.

Figura 2 - Curvas de regressão linear das concentrações plasmáticas de progesterona nas receptoras de embrião bovino gestantes, dos grupos controle e tratado com $10 \mathrm{mg}$ de buserelina, no $4^{\circ} \mathrm{dia}$ após a inovulação, em função dos dias de coleta de sangue.

Figure 2 - Linear regression curves of plasma progesterone concentrations on pregnants bovine embryo recipients of the treated group with $10 \mathrm{mg}$ of buserelin, in the fourth day after embryo transfer and of the control group in function of the blood collection days.

devido ao efeito luteotrópico da buserelina sobre o corpo lúteo e a formação do corpo lúteo acessório em alguns animais tratados. Segundo THATCHER et al. (1989), o aumento no número de folículos "nublados", observado pela ultra-sonografia após o tratamento com $10 \mathrm{mg}$ de buserelina, entre os dias 11 e 13 do ciclo, sugere a formação de folículos luteinizados e produtores de progesterona. Este fato corrobora os achados de RYAN et al. (1994), os quais sugerem que a liberação de hormônio luteinizante $(\mathrm{LH})$ da hipófise anterior, causada pela administração de $10 \mathrm{mg}$ de buserelina no dia 12 do ciclo estral de vacas inseminadas, provavelmente recruta maior número de células da granulosa para a produção de progesterona, acarretando aumento nos níveis séricos de progesterona nas vacas e/ou novilhas tratadas.

O pico de liberação de hormônio luteinizante (LH) foi também observado por STEVENSON et al. (1993) após administração de $8 \mathrm{mg}$ de buserelina entre os dias 11 e 14 do ciclo estral de vacas inseminadas. Estes pesquisadores observaram aumento nos níveis séricos de progesterona do grupo- tratado, o que reforça a hipótese de luteinização de folículos e, assim, aumento na secreção de progesterona, refletindo nos níveis plasmáticos de progesterona do grupo-tratado com buserelina.

\section{Conclusões}

Não se observou efeito da utilização de buserelina sobre o percentual de gestação de receptoras de embrião bovino.

O uso de uma única dose de buserelina aumentou os níveis plasmáticos de progesterona nas receptoras gestantes do grupo tratado em comparação às do grupo controle.

\section{Agradecimento}

Ao professor Jorge Luíz Pesquero do Instituto de Ciências Biológicas-UFMG), pela valiosa colaboração nas análises de radioimunoensaio para progesterona.

Ao professor Og Francisco Fonseca de Souza do Departamento de Biologia Animal-UFV, pelas valiosas orientações na análise estatística dos dados.

À CAPES, pelo fornecimento de bolsa de estudos durante a realização desta pesquisa.

\section{Referências Bibliográficas}

BECK, N.F.G., PETERS, A.R., WILLIAMS, S.P. 1994. The effect of GnRH agonist (buserelin) treatment on day 12 post mating on the reproductive performance of ewes. Br. Soc. Anim. Prod., 58:243-247.

FREI, R.E., SCHULTZ, G.A., CHURCH, R.B. 1989. Qualitative and quantitative changes in protein synthesis occur at the 8 -16-cell stage of embryogenesis in the cow. J. Reprod. and Fert., 86:637-641.

GEISERT, R.D., MORGAN, G.L., SHORT, E.C. et al. 1992. Endocrine events associated with endometrial function and conceptus development in cattle. Reprod. Fert. Develop. 4:301-305.

GOMES, F.P. 1987.Curso de estatística experimental. 7.ed. Piracicaba: Nobel. 430p.

GONG, J.G., BRAMLEY, T.A., GUTIERREZ, C.G. et al. 1995. Effects of chronic treatment with a gonadotrophin - releasing hormone agonist on peripheral concentrations of FSH and LH, and ovarian function in heifers. J. Reprod. Fert., 105:263-270.

HAFEZ, E.S.E. 1992. Reproduction in farm animals. 6.ed. Philadelphia: Lea \& Febiger. 573p.

HANSEN, P.J., EALY, A.D. Effects of heat stress on the establishment and maintenance of pregnancy in cattle. In: CONGRESSO BRASILEIRO DE REPRODUÇÃO ANIMAL, 9, 1991, Belo Horizonte. Anais... Belo Horizonte: CBRA, 1991. p.108-119.

HARVEY, M.J.A., RENTON, J.P., SALAHEDDINE, M. et al. 1994. Ovarian and clinical response of cattle to buserelin. Veterinary Records, 134(2):168-171. 
Rev. bras. zootec.

HOYER, P.B., NISWENDER, G.D. 1986. Adenosine 3', 5'monophosphate-binding capacity in small and large ovine luteal cells. Endocrinology, 119(4):1822-1829.

KALTENBACH, C.C., DUNN, T.G., KISER, T.E. et al. 1974. Release of FSH and LH in beef heifers by synthetic gonadotrophin releasing hormone. J. Anim. Sci., 38(2):357-362.

KENNEDY, L.G., BOLAND, M.P., GORDON, I. 1983. The effect of embryo quality at freezing on subsequent development of thawed cow embryos. Theriogenology, 19(6):823-832.

KESLER, D.J. 1997. Norgestomet implants maintain pregnancy in ovariectomized heifers. Theriogenology, 48:89-98.

LAMMING, G.E., MANN, G.E. 1993. Progesterone concentration affects the development of the luteolytic mechanism in the cow. J. Reprod. Fert., Abstract, 11:8-17.

LAU, T., GOW, G.B., FAIRCLOUGH, R.J. 1992. Differential effects of progesterone treatment on the oxytocin-induced prostaglandin response and the levels of endometrial oxytocin receptors in ovariectomized ewes. Biol. Reprod., 46:17-22.

LAWSON, R.A.S., CAHILL, L.P. 1983. Modification of the embryo-maternal relationship in ewes by progesterone treatment early in the oestrous cycle. J. Reprod. Fert., 67:473-475.

LEWIS, G.S., CALDWELL, D.W., REXROAD JR., C. E. et al. 1990. Effect of gonadotropin-releasing hormone and human chorionic gonadotropin on pregnancy rate in dairy cattle. J. Dairy Sci., 73:66-72.

LINDNER, G.M., WRIGHT JR., R.W. 1983. Bovine embryo morphology and evaluation. Theriogenology, 20(4):407-416.

MACMILLAN, K.L., TAUFA, V.K., DAY, A.M. 1986. Effects of an agonist of gonadotrophin releasing hormone (buserelin) in cattle, III. pregnancy rates after a post-insemination injection during metoestrus or dioestrus. Anim. Reprod. Sci., 11:1-10.

MANN, G.E., LAMMING, G.E. 1995. Effects of treatment with buserelin on plasma concentrations of oestradiol and progesterone and cycle length in the cow. Br. Vet. J., 151(4):427-432.

MANN, G.E., LAMMING, G.E., FRAY, M.D. 1995. Plasma oestradiol and progesterone during early pregnancy in the cow and the effects of treatment with buserelin. Anim. Reprod. Sci., 37:121-131.

MILVAE, R.A., MURPHY, B.D., HANSEL, W. 1984. Prolongation of bovine estrous cycle with a gonadotropin- releasing hormone analog. Biol. Reprod., 31:664-670.

NAKAO, T., NARITA, S., TANAKA, K. et al. 1983. Improvement of first-service pregnancy rate in cows with gonadotropin-releasing hormone analog. Theriogenology, 20:(1)111-119.

NISWENDER, G.D., NETT, T.M. 1988. The corpus luteum and its control. In: KNOBIL E., NEILL J. (Eds.) The physiology of reproduction. New York: Raven Press. p.489-525.

REMSEM, L.G., ROUSSEL, J.D., KARIHALOO, A.K. 1982. Pregnancy rates relating to plasma progesterone levels in recipient heifers at day of transfer. Theriogenology, 18(3):365-372.

RETTMER, I., JEFFREY, S.S., LARRY, R.C. 1992. Endocrine responses and ovarian changes in inseminated dairy heifers after an injection of a GnRH agonist 11 to 13 days after estrus. J. Anim. Sci., 70:508-517.

ROBERTS, R.M., XIE, S., MATHIALAGAN, N. 1996. Maternal recognition of pregnancy. Biol. Reprod., 54:294-302.

RODRIGUES, J.L. Aspectos da congelação de embriões bovinos. In: REUNIÃO ANUAL DA SOCIEDADE BRASILEIRA DE TRANSFERÊNCIA DE EMBRIÕES, 7, 1992, Jaboticabal. Anais... Jaboticabal: SBTE, 1992. p.55-79.
RUMPF, R., de BEM, A.R., SOUZA, R.V. et al. Bissecção de embriões de bovinos. In: REUNIÃO ANUAL DA SOCIEDADE BRASILEIRA DE TRANSFERÊNCIA DE EMBRIÕES, 7, 1992, Jaboticabal. Anais... Jaboticabal: SBTE, 1992. p.92-113.

RYAN, D.P., SNIJDERS, S., CONDON, T. et al. 1994. Endocrine and ovarian responses and pregnancy rates in dairy cows following the administration of a gonadotropin releasing hormone analog at the time of artificial insemination or at midcycle post insemination. Anim. Reprod. Sci., 34:179-191.

SHELDON, I.M., DOBSON, H. 1993. Effects of gonadotrophin releasing hormone administered 11 days after insemination on the pregnancy rates of cattle to the first and later services. Veterinary Record, 133:160-163.

SREENAN, J.M., DISKIN, M.G. 1987. Factors affecting pregnancy rate following embryo transfer in the cow. Theriogenology, 27(1):99-113.

STEVEN, J.O. 1983. Clinical uses of luteinizing hormone-releasing hormone. Fertility and Sterility, 39(5):577-590.

STEVENSON, J.S., PHATAK, A.P., RETTMER, I. et al. 1993. Postinsemination administration of receptal: follicular dynamics, duration of cycle, hormonal responses, and pregnancy rates. J. Dairy Sci., 76:2536-2547.

THATCHER, W.W., MACMILLAN, K.L., HANSEN, P.J. et al. 1989. Concepts for regulation of corpus luteum function by the conceptus and ovarian follicles to improve fertility. Theriogenology, 31(1):149-164.

TWAGIRAMUNGU, H., GUILBAULT, L.A., PROULX, J. et al. 1992. Synchronization of estrus and fertility in beef cattle with two injections of buserelin and prostaglandin. Theriogenology, 38:1131-1144.

UNIVERSIDADE FEDERAL DE VIÇOSA - UFV. 1997. SAEG - Sistemas de análises estatísticas e genéticas. Versão 7.1.Viçosa, MG. 150p.(Manual do usuário).

VALLET, J.L., LAMMING, G.E. 1991. Ovine conceptus secretory proteins and bovine recombinant interferon decrease endometrial oxytocin receptor concentrations in cyclic and progesterone treated ovariectomized ewes. J. Endocrinology, 131:475-482.

WHITTINGHAM, D.G. 1971. Survival of mouse embryos after freezing and thawing. Nature, 233:125-132.

WILDMAN, E.E., JONES, G.M., WAGNER, P.E. et al. 1982 A dairy cow body condition scoring system and its relationship to selected production characteristics. J. Dairy Sci., 65(3):495-501.
Recebido em: 15/07/99

Aceito em: 11/12/00 